\title{
ChromagarTM Col-Apse for Detection of Colistin Resistance in Clinical Isolates of Multidrug Resistant Gram Negative Bacilli
}

\author{
Saad Ali, Wajid Hussain, Farooq Ahmed, Raja Kamran Afzal, Irfan Mirza and Umer Khurshid \\ Department of Microbiology, Armed Forces Institute of Pathology (AFIP), Rawalpindi, Pakistan
}

\begin{abstract}
:
Objective: To determine the diagnostic accuracy of CHROMagar ${ }^{\top M}$ COL-APSE, for detection of colistin resistance in clinical isolates of multi drug resistant (MDR) gram negative bacilli (GNB).

Study Design: Cross-sectional validation study.

Place and Duration of Study: Department of Microbiology, Armed Forces Institute of Pathology (AFIP), Rawalpindi, Pakistan from February 2019 to August 2019.

Methodology: Total 96 MDR-GNB in clinical isolates were included. Isolates were identified using gram stain, catalase, oxidase, API 20E and API 20NE. After taking approval from Institutional Ethical Review Committee, colistin susceptibility was determined simultaneously by CHROMagar ${ }^{\text {TM }}$ COL-APSE (using $1 \times 10^{5} \mathrm{CFU} / \mathrm{ml}$ inoculum) and Broth Micro Dilution (BMD) Minimum Inhibitory Concentration (MIC) method as per CLSI. EUCAST guidelines were followed for interpretation of susceptibility profile. Results were validated with gold standard test, i.e. BMD.

Results: Out of 96 MDR clinical isolates, the distribution was $K$. pneumoniae $\mathrm{n}=63, E$. coli $\mathrm{n}=18$, A. baumannii $\mathrm{n}=11, \mathrm{C}$. freundii $\mathrm{n}=3$, and $E$. cloacae $\mathrm{n}=1$. The sensitivity, specificity, positive predictive value, negative predictive value and diagnostic accuracy of CHROMagar ${ }^{\mathrm{TM}}$ COL-APSE for detection of colistin resistance, keeping BMD-MIC method as gold standard, was $97.96 \%$, $97.87 \%, 97.96 \%, 97.87 \%$ and $97.92 \%$, respectively.

Conclusion: $\mathrm{CHROMagar}{ }^{\mathrm{TM}}$ COL-APSE can be used as screening agar by direct streaking of specimen as well as diagnostic for detection of colistin resistance by the use of standardised inoculum. It can be used as a critical time saving method for colistin resistance detection in absence of expertise or manpower required for BMD as well as the cost required for genetic sequencing to detect MCR genes.
\end{abstract}

Key Words: Multi drug resistant (MDR), Gram negative bacilli (GNB), Colistin resistance, CHROMagar ${ }^{T M}$ COL-APSE.

How to cite this article: Ali S, Hussain W, Ahmed F, Afzal RK, Mirza I, Khurshid U. ChromagarTM Col-Apse for Detection of Colistin Resistance in Clinical Isolates of Multidrug Resistant Gram Negative Bacilli. J Coll Physicians Surg Pak 2022; 32(02):177-180.

\section{INTRODUCTION}

Multi drug resistance (MDR) in bacteria not only poses a threat to patient, but also creates problems for treating physicians, especially in intensive care setups, where due to comorbid various options available for MDR organisms, may not be beneficial. ${ }^{1}$ Gram negative bacilli (GNB) are among the common causes of healthcare as well as community acquired infections with a high propensity for antimicrobial resistance. ${ }^{2}$ Alarmingly, the tap of antibiotic development has run dry with the last major antibiotic class discovery in 1990s. ${ }^{3}$

Correspondence to: Dr. Saad Ali, Department of Microbiology, Armed Forces Institute of Pathology (AFIP),

Rawalpindi, Pakistan

E-mail: alii.saad@live.com

Received: October 11, 2021; Revised: November 12, 2021;

Accepted: December 10, 2021

DOI: https://doi.org/10.29271/jcpsp.2022.02.177
On the other hand, resistance to all currently available classes of antibiotics is rising exponentially, which has led to the use of older antibiotics like colistin and fosfomycin. Earlier, they were put out of use due to toxicity and lack of studies, as there were much safer options available. The combined effect of these two phenomena have brought colistin back as a crucial part of management against MDR-GNB and it is now being routinely advised as a last option. ${ }^{4}$ As a consequence, there has been a rapid rise in resistance to colistin as well, especially with the plasmid mediated carbapenem and colistin resistance. It leaves with no other option for management; and remarkably denotes the start of post-antibiotic era. ${ }^{5}$ According to a recent study conducted in Karachi, frequency of colistin resistance is found to be $15.9 \%$ in carbapenem resistant enterobacterales isolates. ${ }^{6}$

The solution to this crisis lies on accurate diagnostics, wise infectious disease management, and proper infection control measures.

There should be timely identification of colistin-resistant isolates and manage them efficiently. ${ }^{7}$ Due to the lack of 
studies, a routine, quick and accurate method for colistin susceptibility is yet to be developed. Currently Broth Micro Dilution (BMD) is the recommended method for detection of Minimum Inhibitory Concentration (MIC); but there are issues, like adsorption to plastic and adjustment of cations. ${ }^{8}$ On the other hand, the diffusion of colistin molecule in agar is yet to be studied, due to which disk diffusion is not recommended method. ${ }^{9}$ BMD, apart from its limitations, is a long and critical procedure which requires expertise, long time procedure itself, as well as incubation with a difficult interpretation at the end, which may at times lead to false positive or negative due to observererror. ${ }^{10}$

Indicators have been used in agar medium for long time; but in recent years, chromogenic media has provided a better and rapid approach in identifying as well as differentiating organisms on the basis of growth on agar. ${ }^{11}$

CHROMagar ${ }^{\mathrm{TM}}$ COL-APSE was introduced in 2017 for the surveillance of colistin resistant bacterial pathogens with a lower limit of detection of $10 \mathrm{CFU} / \mathrm{ml} .{ }^{1}$ It yields different colored colonies specific to species. It is a very helpful aid for diagnosing critical colistin resistance by reducing time to detection with minimal expertise.

The aim of this study was to determine the diagnostic accuracy of CHROMagar ${ }^{\mathrm{TM}} \mathrm{COL}-\mathrm{APSE}$ for identification of colistin resistant GNB to help in early prevention of treatment failures in critical patients by rapid detection of colistin resistance. This will add to the ease of performance and cost effectiveness especially in resource limited setups of labs.

\section{METHODOLOGY}

This cross-sectional validation study was carried out at the Department of Microbiology, Armed Forces Institute of Pathology (AFIP), Rawalpindi from February 2019 to August 2019. Sample size of 96 was calculated, using sensitivity specificity calculator keeping prevalence of colistin resistance at $15.9 \%{ }^{6,12}$ Non-probability consecutive sampling was done. Permission of this study was obtained from Institutional Ethical Review Board.

To evaluate the diagnostic accuracy of the CHROMagar ${ }^{\mathrm{TM}} \mathrm{COL}$ APSE, inclusion criteria was set as MDR organisms having resistance to single agent of three or more antimicrobial classes. ${ }^{13}$ Same isolate from duplicate samples of same patients were excluded. These isolates were identified on the basis of colony morphology, gram stain, catalase, oxidase, API 20E, and API 20NE (BioMérieux).

To determine colistin MICs for these isolates, reference BMD method in Cation-Adjusted Mueller Hinton broth (CAMB) as recommended by Clinical Laboratory Standard Institute (CLSI), 2019 guidelines was used. ${ }^{14,15}$ Isolates with colistin MICs $\leq 2$ $\mu \mathrm{g} / \mathrm{mL}$ were categorised as sensitive, and those with MICs $\geq 4$ $\mu \mathrm{g} / \mathrm{mL}$ as resistant as per EUCAST guidelines (2019). ${ }^{16}$ For BMD, 96 well-microtiter plates were inoculated with 10 isolates and 2 controls, both positive and negative for colistin resistance, on each plate. Each plate had sterility and growth controls as well. Colistin Sulfate powder (Sigma-Aldrich) was used to make antimicrobial solution of $32 \mathrm{ug} / \mathrm{ml}$ concentration, which was diluted two-folds over 6 wells from $16 \mathrm{ug} / \mathrm{ml}$ to $0.5 \mathrm{ug} / \mathrm{ml}$. Each well was inoculated with $0.05 \mathrm{ml}$ of antimicrobial agent except growth control well. Isolate inoculum was prepared to a final density of $5 \times 10^{5} \mathrm{CFU} / \mathrm{ml}$ and $0.05 \mathrm{ml}$ was dispensed in each well except sterility well. This inoculum was also applied on a quadrant of blood agar and MacConkey agar to rule out any contamination during this procedure.

CHROMagar $^{\mathrm{TM}}$ COL-APSE plates were prepared, according to manufacturer's instructions, and stored at $2-8^{\circ} \mathrm{C}$. These plates were allowed to warm at room temperature before inoculation. Selected MDR isolates were processed by direct streaking on the plates. The plates were incubated in aerobic conditions at $37^{\circ} \mathrm{C}$ for $18-24$ hours. Any growth was considered as positive (Colistin resistant isolate) and no growth as negative (Colistin sensitive isolate). Initially on application of $0.5 \mathrm{McF}$ arland standard inoculum ( $3 \times 10^{8} \mathrm{CFU} / \mathrm{ml}$ ), it was observed that colistin susceptible isolates were able to grow on $\mathrm{CHROMagar}^{\mathrm{TM}} \mathrm{COL}$ APSE (Figure 1a).

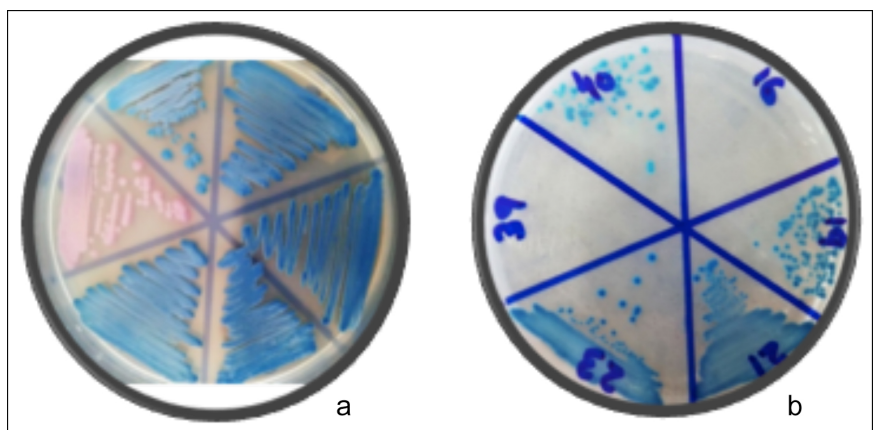

Figure 1: (a) Before standardising inoculum (b) After standardising inoculum.

Inoculum was diluted to a density of $1 \times 10^{5} \mathrm{CFU} / \mathrm{ml}$, following the principle of agar dilution as per CLSI guidelines, which led to growth concordant with results of BMD (Figure 1b). So for, in whole study a standard density inoculum of $1 \times 10^{5} \mathrm{CFU} / \mathrm{ml}$ was used.

The data obtained was entered in SPSS (version 25). Diagnostic parameters were calculated by analysis of descriptive data using bivariate cross tabulation and significance was ascertained by chi square test and Pearson equation.

\section{RESULTS}

Out of 96 isolates, the distribution was given in Figure 2. CHROMagar $^{\top \mathrm{M}}$ COL-APSE was able to identify all colistin resistant isolates as per product manual. Among these isolates, colistin resistance was found in $76 \%$ of $K$. pneumoniae while $0.9 \%$ in $A$. baumannii. Isolates of $E$. Coli, C. freundii and E. cloacae were all found to be sensitive to colistin in this study.

All the isolates were subjected to CHROMagar ${ }^{T M}$ COL-APSE test and 48 were found to be true positive while one was false positive. Among 47, CHROMagar ${ }^{\mathrm{TM}} \mathrm{COL}-\mathrm{APSE}$ negative patients, one 
(false negative) had colistin resistance on BMD method whereas 46 (true negative) had no colistin resistance on BMD method.

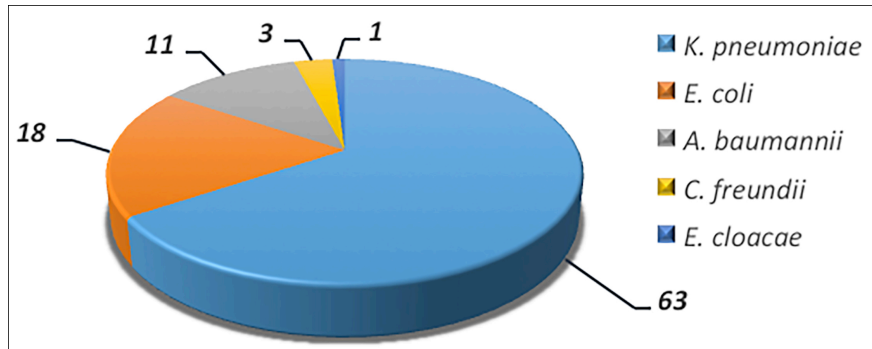

Figure 2: Distribution of clinical isolates.

Overall sensitivity, specificity, positive predictive value, negative predictive value and diagnostic accuracy of CHROMagarTM COL-APSE, for detection of Colistin resistance in clinical isolates of multidrug resistant GNB, keeping BMD method as gold standard was $97.96 \%, 97.87 \%, 97.96 \%, 97.87 \%$ and $97.92 \%$, respectively.

\section{DISCUSSION}

Since the resistance against colistin is at a rise, it is essential to formulate a method which is accurate as well as easy to perform for prompt diagnosis and management of such resistant bacteria. ${ }^{17}$ Chromogenic media has the ability to differentiate multiple species on the basis of colors simultaneously which could not be possible with indicators.

CHROMagar $^{\mathrm{TM}}$ COL-APSE is a chromogenic media that is affected by the inoculum density. This was noted in this study as well, that on standardising the inoculum to $1 \times 10^{5} \mathrm{CFU} / \mathrm{mICHRO-}$ Magar ${ }^{\mathrm{TM}} \mathrm{COL}-\mathrm{APSE}$ washighly sensitive and specific.

In a study conducted by Yoshimasa et al., for dissemination colistin resistant $E$. coli in healthy residents of Vietnam sensitivity of CHROMagar $^{\mathrm{TM}}$ COL-APSE was found to be $100 \%$; however, specificity was low as compared to this study at $68 \% .{ }^{18}$ This could be probably due to issue related to inoculum density as in this study all growth was taken as colistin resistant.

Momin et al. conducted a study for evaluation of CHROMagar ${ }^{\mathrm{TM}}$ COL-APSE for isolation and differentiation of GNB. In this study, inoculum was standardised to $10^{5} \mathrm{CFU} / \mathrm{ml}$ and further $10 \mathrm{UL}$ drop was inoculated on CHROMagar ${ }^{\mathrm{TM}}$ COL-APSE, which yielded sensitivity and specificity of $100 \%{ }^{19}$ It is comparable to the present study as with similar inoculum density similar results were achieved.

$\mathrm{CHROMID}^{\circledR}$ colistin R agar is another chromogenic media that has been available for detection of colistin resistant enterobacterales; but its evaluation yielded a sensitivity of $88.1 \%$, lower than that of CHROMagar COL-APSE ${ }^{\mathrm{TM}}$ that is $97.96 \%$ in this study; but with a higher specificity of $100 \%{ }^{20}$

However, the limitations of $\mathrm{CHROMID}^{\mathrm{R}}$ colistin $\mathrm{R}$ agar are that it needs pre enrichment of 4-5 hours and is recommended to be used in stool and rectal swab specimens.

\section{CONCLUSION}

The diagnostic accuracy of CHROMagar ${ }^{\mathrm{TM}}$ COL-APSE, for detection of colistin resistance in clinical isolates of multidrug resistant, GNB is excellent. It can be used as diagnostic tool by inoculating standardised density of organism.

\section{ETHICALAPPROVAL:}

This study was approved by the Institutional Review Board of AFIP, Rawalpindi, Pakistan.

\section{PATIENTS' CONSENTS:}

Informed consents were taken from all the participants, whom samples were included in the study.

\section{CONFLICT OF INTEREST:}

The authors declared no conflict of interest.

\section{AUTHORS' CONTRIBUTION:}

SA: Microbiology research work, patient data and sample collection, article writing, statistics.

WH: Supervision of project, article writing, review of article.

FA: Data collection, microbiology work, article writing.

RKA: Article review, discussion writing.

IAM: Discussion review, article review.

UK: Statistics, variable correlation, review article.

\section{REFERENCES}

1. Ruppé É, Woerther PL, Barbier F. Mechanisms of antimicrobial resistance in Gram-negative bacilli. Ann Intensive Care 2015; 5(1):21. doi: 10.1186/s13613015-0061-0.

2. van Duin $D$, Paterson $D L$. Multidrug-resistant bacteria in the community: Trends and lessons learned. Infect Dis Clin North Am 2016; 30(2):377-90. doi: 10.1016/j.idc.2016. 02.004.

3. Lewis K. The science of antibiotic discovery. Cell 2020; 181(1):29-45. doi: 10.1016/j.cell.2020.02.056.

4. Catry B, Cavaleri M, Baptiste K, Grave K, Grein K, Holm A, et al. Use of Colistin-containing products within the European Union and European Economic Area (EU/EEA): development of resistance in animals and possible impact on human and animal health. Int J Antimicrob Agents 2015; 46(3):297-306. doi: 10.1016/j.ijantimicag.2015.06.005.

5. Osei Sekyere J, Govinden U, Bester LA, Essack SY. Colistin and tigecycline resistance in carbapenemase-producing Gram-negative bacteria: Emerging resistance mechanisms and detection methods. J Appl Microbiol 2016; 121(3): 601-17. doi: 10.1111/jam.13169.

6. Qamar S, Shaheen N, Shakoor S, Farooqi J, Jabeen K, Hasan R. Frequency of Colistin and fosfomycin resistance in carbapenem-resistant Enterobacteriaceae from a tertiary care hospital in Karachi. Infect Drug Resist 2017; 10:231-6. doi: 10.2147/IDR.S136777.

7. Xavier BB, Lammens C, Ruhal R, Kumar-Singh S, Butaye P, Goossens $\mathrm{H}$, et al. Identification of a novel plasmidmediated Colistin-resistance gene, mcr-2, in Escherichia coli, Belgium, June 2016. Euro Surveillance Monthly 2016; 21(27):30280. doi: 10.2807/1560-7917.ES.2016.21.27. 
30280.

8. Ezadi F, Ardebili A, Mirnejad R. Antimicrobial susceptibility testing for polymyxins: Challenges, issues, and recommendations. J Clin Microbiol 2019; 57(4). doi: 10.1128/JCM.01390-18.

9. Nation RL, Li J, Cars O, Couet W, Dudley MN, Kaye KS, et al. Framework for optimisation of the clinical use of Colistin and polymyxin B: The prato polymyxin consensus. Lancet Infect Dis 2015; 15(2):225-34. doi: 10.1016/S14733099(14)70850-3.

10. Simar S, Sibley D, Ashcraft D, Pankey G. Colistin, Colistin B. Minimal inhibitory concentrations determined by etest found unreliable for Gram-negative bacilli. Ochsner J 2017; 17(3):239-42.

11. Perry JD, Freydiere AM. The application of chromogenic media in clinical microbiology. J Appl Microbiol 2007; 103(6):2046-55. doi: 10.1111/j.1365-2672.2007.03442.x.

12. Buderer NMF. Statistical methodology: I. In corporating the prevalence of disease into the sample size calculation for sensitivity and specificity. Acad Emerg Med 1996; 3(9): 895-900. doi: 10.1111/j.1553-2712.1996.tb03538.x.

13. Magiorakos AP, Srinivasan A, Carey RT, Carmeli $Y$, Falagas MT, Giske CT, et al. Multidrug-resistant, extensively drugresistant and pandrug-resistant bacteria: An international expert proposal for interim standard definitions for acquired resistance. Clin Microbiol Infect 2012; 18(3): 268-81. doi: 10.1111/j.1469-0691. 2011.03570.x. Epub 2011 Jul 27.

14. Clinical and laboratory standards institute. Methods for dilution antimicrobial susceptibility tests for bacteria that grow aerobically, 9th ED, vol. 32. Wayne, Pennsylvania, USA: CLSI; 2012. pp. M07-A9.

15. Clinical and laboratory standards institute. Performance standards for antimicrobial susceptibility testing; supplement M100S, 25th ed. Wayne, Pennsylvania, USA: CLSI; 2015.

16. The European Committee on antimicrobial susceptibility testing. Breakpoint tables for interpretation of MICS and zone diameters, version 9.0, 2019.

17. Vasoo S. Susceptibility testing for the polymyxins: Two steps back, three steps forward? J Clin Microbiol 2017; 55(9):2573-82. doi: 10.1128/JCM.00888-17.

18. Yamamoto Y, Kawahara R, Fujiya Y, Sasaki T, Hirai I, Khong DT, et al. Wide dissemination of Colistin-resistant Escherichia coli with the mobile resistance gene mcr in healthy residents in Vietnam. J Antimicrob Chemother 2019; 74(2):523-4. doi: 10.1093/jac/dky435.

19. Momin MH, Bean DC, Hendriksen RS, Haenni M, Phee LM, Wareham DW. CHROMagar COL-APSE: A selective bacterial culture medium for the isolation and differentiation of colistin-resistant gram-negative pathogens. J Med Microbiol 2017; 66(11):1554-61. doi: 10.1099/jmm.0.000602.

20. García-Fernández S, García-Castillo M, Ruiz-Garbajosa P, Morosini MI, Bala Y, Zambardi G, et al. Performance of CHROMID ${ }^{\circledR}$ Colistin R agar, a new chromogenic medium for screening of colistin-resistant enterobacterales. Diagn Microbiol Infec Dis 2019; 93(1):1-4. doi: 10.1016/j. diagmicrobio.2018.07.008. 